\title{
Galaxies in southern bright star fields $s^{\star} \star \star$
}

\section{Near-infrared imaging}

\author{
Andrew J. Baker ${ }^{1}$, Richard I. Davies ${ }^{1}$, M. D. Lehnert ${ }^{1}$, N. A. Thatte ${ }^{1,2}$, W. D. Vacca ${ }^{1}$, O. R. Hainaut ${ }^{3}$, M. J. Jarvis ${ }^{2,4}$, \\ G. K. Miley ${ }^{4}$, and H. J. A. Röttgering ${ }^{4}$
}

1 Max-Planck-Institut für extraterrestrische Physik, Postfach 1312, 85741 Garching, Germany

e-mail: [ajb, davies, mlehnert, thatte, vacca]@mpe.mpg.de

2 University of Oxford Astrophysics, Keble Road, Oxford OX1 3RH, UK

e-mail: mjj@astro.ox.ac.uk

${ }^{3}$ European Southern Observatory, Alonso de Cordova 3107, Casilla 19001, Vitacura, Santiago, Chile e-mail: ohainaut@eso.org

4 Sterrewacht Leiden, Postbus 9513, 2300 RA Leiden, The Netherlands

e-mail: [miley,rottgeri]@strw.leidenuniv.nl

Received 7 March 2003 / Accepted 27 May 2003

\begin{abstract}
As a prerequisite for cosmological studies using adaptive optics techniques, we have begun to identify and characterize faint sources in the vicinity of bright stars at high Galactic latitudes. The initial phase of this work has been a program of $K_{\mathrm{s}}$ imaging conducted with SOFI at the ESO NTT. From observations of 42 southern fields evenly divided between the spring and autumn skies, we have identified 391 additional stars and 1589 galaxies lying at separations $\Delta \theta \leq 60^{\prime \prime}$ from candidate guide stars in the magnitude range $9.0 \leq R \leq 12.4$. When analyzed as a "discrete deep field" with $131 \mathrm{arcmin}^{2}$ area, our dataset gives galaxy number counts that agree with those derived previously over the range $16 \leq K_{\mathrm{s}}<20.5$. This consistency indicates that in the aggregate, our fields should be suitable for future statistical studies. We provide our source catalogue as a resource for users of large telescopes in the southern hemisphere.
\end{abstract}

Key words. instrumentation: adaptive optics - galaxies: photometry - infrared: galaxies - infrared: stars

\section{Introduction}

Understanding the mechanisms that drive galaxy formation and evolution is a central objective of modern astrophysics. While the structures and dynamics of nearby galaxies constitute a valuable fossil record, these are much less informative than the properties of galaxies observed at the epochs of their formation and early evolution. By studying galaxies' structures, dynamics, fundamental scaling laws, and distributions and rates of star formation over a range in redshift, it is possible to trace the evolving role and subtle interactions of key physical processes such as feedback, mass loss, merging, and secular evolution, among others. Disentangling these processes in nearby systems

Send offprint requests to: A. J. Baker,

e-mail: ajb@mpe.mpg.de

* Based on observations obtained at the European Southern Observatory, Chile, for programmes 66.A-0361 and 68.A-0440.

$\star \star$ The entirety of Table 3 is only available in electronic form at the CDS via anonymous ftp to

cdsarc.u-strasbg.fr $(130.79 .128 .5)$ or via

http://cdsweb.u-strasbg.fr/cgi-bin/qcat?J/A+A/406/593 is already extremely difficult. Attempts to extend this effort to newly-formed galaxies require data whose high spatial resolution and dynamic range can only be achieved by $8 \mathrm{~m}$-class telescopes using adaptive optics (AO) techniques to work at or near their diffraction limits.

AO relies on the detection of, and compensation for, the distortions of wavefronts from a bright pointlike object. For science targets that are themselves too faint to permit wavefront sensing, or too diffuse to sustain efficient wavefront correction (see Rousset 1994), a bright ( $V \leq 13$ ), nearby $\left(\Delta \theta \leq 30^{\prime \prime}\right)$ natural guide star (NGS) is required. Exactly how bright and nearby depends on the local atmospheric conditions; the above values correspond to the typical regime in which the wavefront sensor is not photon-limited and the off-axis Strehl ratio at $2.2 \mu \mathrm{m}$ is not too severely reduced (e.g., Le Louarn et al. 1998). In the absence of a nearby NGS, higher-order wavefront corrections using a laser guide star (LGS) can suffice, provided a moderately bright $(V \sim 16)$ reference star lies relatively close $\left(\Delta \theta \leq 90^{\prime \prime}\right)$ to the science target for determination of the lowest-order tiptilt correction. Switching from LGS to NGS mode trades a gain 


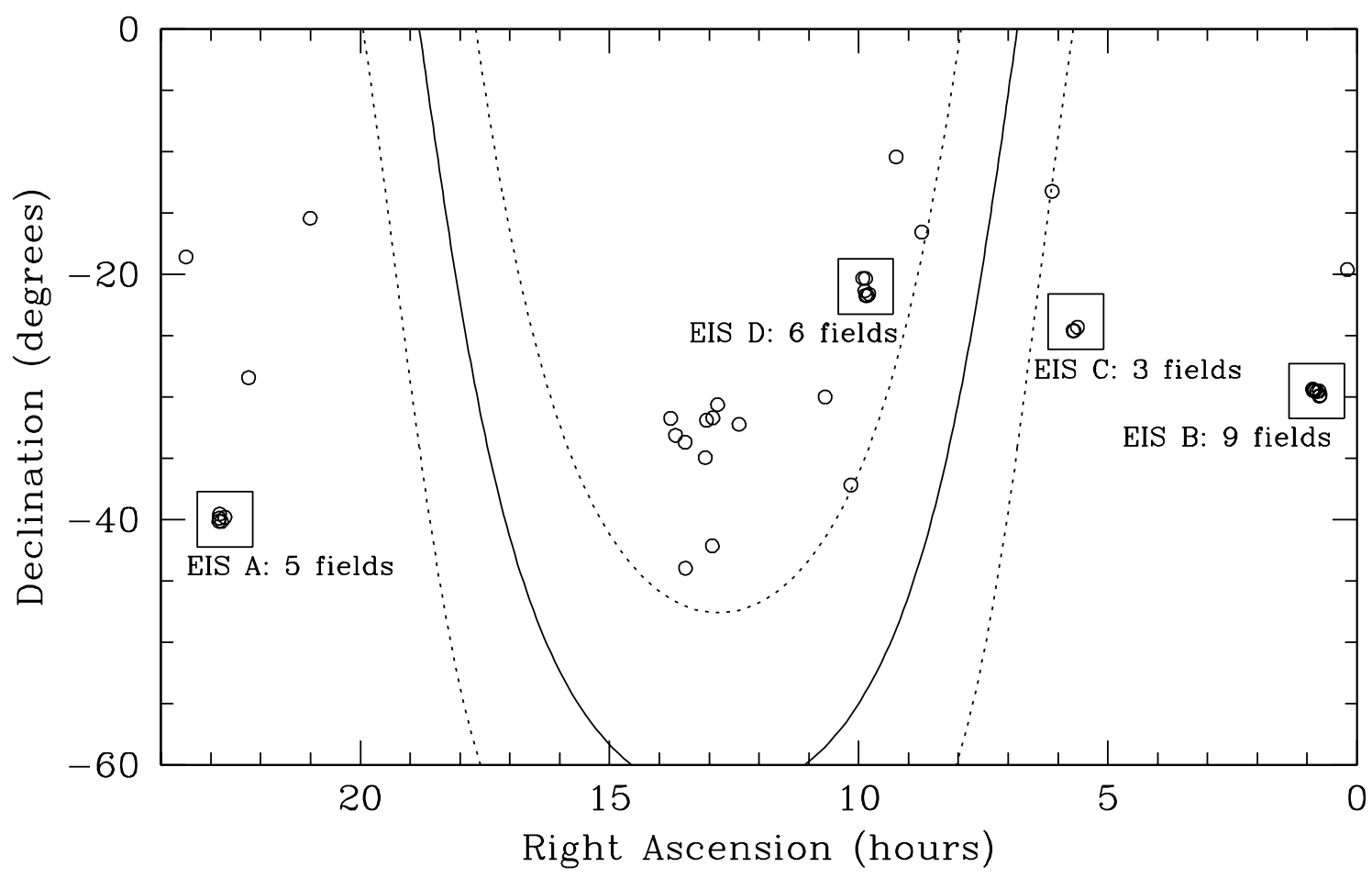

Fig. 1. Positions of the 42 southern bright star fields observed in this survey, denoted by open circles. The solid line shows the Galactic plane; the dotted lines to either side indicate Galactic latitude $|b|=15^{\circ}$. The four EIS Wide patches from which some of the fields were selected are indicated by boxes (not drawn to scale).

in sky coverage for a loss in the highest possible Strehl ratio, however, since the LGS samples atmospheric turbulence in a conical (rather than cylindrical) volume. The phase error due to such focus anisoplanatism increases with mirror diameter as $D^{5 / 6}$ (e.g., Le Louarn et al. 1998, and references therein). Until the advent of multiple-LGS systems that can correct for this effect (e.g., Tallon \& Foy 1990), AO systems on 8 m-class telescopes will therefore reach the highest Strehl ratios only when operating in NGS mode.

In order to conduct cosmological studies with the present generation of AO technology (e.g., Larkin et al. 2000; Davies et al. 2001; Glassman et al. 2002; Davies et al. 2003), it is necessary to identify distant galaxies in the vicinity of bright guide stars. Unfortunately, most existing surveys either avoid bright stars (e.g., the Hubble Deep Field), or like DENIS and 2MASS (Epchtein et al. 1997; Skrutskie et al. 1997) are too shallow to detect very many targets at cosmological distances. We have therefore begun a program to characterize the extragalactic sources lying close to bright stars at high Galactic latitudes. Similar work underway by other authors (Larkin \& Glassman 1999) has focused on fields easily observable from the Keck telescopes; our targets are specifically chosen to be at southern declinations suitable for observations with the NAOS-CONICA (Rousset et al. 1998; Lenzen et al. 1998) and SINFONI (Thatte et al. 1998) instruments on the ESO Very Large Telescope (VLT).

The initial phase of our program has been a campaign of (non-AO-assisted) near-IR imaging, since information about source magnitudes and surface brightness profiles will be most useful at the wavelength of eventual AO operation. For deep imaging studies in particular, the availability of seeing-limited data for comparison will allow the empirical determination of the surface brightness selection effects influencing diffractionlimited data (e.g., whether a given faint "point" source is only the nucleus of an unremarkable extended galaxy). Blind diffraction-limited imaging of random fields, in contrast, remains vulnerable to unknown biases and uncertainties in the surface densities of true point sources. We have already followed up our near-IR imaging with optical imaging and multiobject spectroscopy of many of our fields; discussion of these data is deferred to future papers.

\section{Observations and data reduction}

\subsection{Target selection}

Table 1 lists the identifications, coordinates, and magnitudes of the 42 bright stars that define our near-IR imaging sample; Fig. 1 shows their distribution on the sky. The majority of these targets were selected from the USNO-A2.0 catalogue (Monet et al. 1998) according to criteria designed to ensure optimal performance of a near-IR science instrument assisted by a visible-wavelength (e.g., $0.45-1.0 \mu \mathrm{m}$ for NAOS-CONICA) wavefront sensor:

1. magnitude $10.3 \leq R \leq 12.4$, i.e., bright enough in the visible for wavefront sensing to be robust;

2. color $B-R \leq 1.1$, i.e., bluer than that of a type G1 star, in order to maximize the number of photons on the wavefront sensor while minimizing the amount of near-IR light scattered onto the science detector; 
Table 1. Bright star fields. Columns are (1) field identifier by which the sources in Table 3 are indexed; (2) USNO-B1.0 designation for the bright star (Monet et al. 2003); (3) \& (4) USNO-B1.0 J2000.0 coordinates of the bright star, accurate to $\pm 0.2^{\prime \prime}$; (5) \& (6) USNO-B1.0 proper motions of the bright star in mas $\mathrm{yr}^{-1}$, relative to the YS4.0 reference frame described by Monet et al. (2003); (7) \& (8) USNO-B1.0 B and $R$ magnitudes of the bright star, averaged over two epochs and accurate to $\pm 0.3 \mathrm{mag}$; (9) $K_{\mathrm{s}}$ magnitude of the bright star, from our SOFI imaging; (10) date(s) on which the field was observed with SOFI, as indexed in Table 2; (11) total SOFI integration time, in minutes; (12) note as to whether the field falls inside an EIS Wide patch or near an NVSS source.

\begin{tabular}{|c|c|c|c|c|c|c|c|c|c|c|c|}
\hline $\begin{array}{l}\text { Field } \\
\text { (1) }\end{array}$ & $\begin{array}{c}\text { USNO-B } 1.0 \\
\text { (2) }\end{array}$ & $\begin{array}{c}\alpha(\mathrm{J} 2000.0) \\
(3)\end{array}$ & $\begin{array}{c}\delta(\mathrm{J} 2000.0) \\
(4)\end{array}$ & $\begin{array}{r}\mu(\alpha) \\
(5)\end{array}$ & $\begin{array}{r}\mu(\delta) \\
(6)\end{array}$ & $\begin{array}{r}B \\
(7)\end{array}$ & $\begin{array}{r}R \\
(8)\end{array}$ & $\begin{array}{l}K_{\mathrm{s}} \\
(9)\end{array}$ & $\begin{array}{l}\text { Night(s) } \\
\text { (10) }\end{array}$ & $\begin{array}{c}\Delta t \\
(11)\end{array}$ & $\begin{array}{l}\text { Note } \\
(12)\end{array}$ \\
\hline SBSF 01 & $0703-0002281$ & 001138.84 & 193711.0 & +8 & -6 & 12.1 & 10.0 & 7.80 & 6 & 30 & NVSS \\
\hline SBSF 02 & 0601-0007980 & 004431.88 & -295230.3 & +24 & -8 & 11.9 & 11.1 & 10.09 & 6 & 30 & EIS B \\
\hline SBSF 03 & 0600-0008265 & 004520.62 & -295646.0 & +32 & +6 & 12.9 & 12.1 & 11.11 & 6 & 30 & EIS B \\
\hline SBSF 04 & 0604-0008038 & 004528.05 & -293140.1 & -12 & +4 & 13.2 & 12.4 & 11.33 & 45 & 20 & EIS B \\
\hline SBSF 05 & 0604-0008436 & 004811.23 & -293414.2 & +92 & -60 & 12.7 & 11.6 & 10.11 & 6 & 20 & EIS B \\
\hline SBSF 06 & 0605-0009459 & 005034.70 & -292632.0 & 0 & 0 & 12.6 & 11.0 & 9.90 & 45 & 30 & EIS B \\
\hline SBSF 07 & 0604-0008993 & 005202.66 & -293048.1 & +24 & +40 & 13.0 & 11.7 & 10.90 & 45 & 30 & EIS B \\
\hline SBSF 08 & 0605-0009658 & 005218.88 & -292717.8 & -12 & -12 & 13.1 & 11.2 & 10.81 & 45 & 30 & EIS B \\
\hline SBSF 09 & 0606-0009140 & 005317.20 & -292229.0 & -2 & -26 & 12.2 & 11.4 & 10.21 & 6 & 30 & EIS B \\
\hline SBSF 10 & $0605-0009783$ & 005317.95 & -292522.6 & +26 & -42 & 12.0 & 11.1 & 9.97 & $J$ & 30 & EIS B \\
\hline SBSF 11 & 0656-0061729 & 053633.64 & -241956.5 & +4 & -10 & 11.8 & 11.1 & 10.08 & 45 & 30 & EIS C \\
\hline SBSF 12 & 0653-0066004 & 054037.80 & -243633.8 & +2 & -20 & 12.1 & 11.5 & 10.26 & 456 & 30 & EIS C \\
\hline SBSF 13 & 0654-0065981 & 054149.93 & -243532.2 & 0 & 0 & 10.9 & 10.8 & 10.46 & 56 & 20 & EIS C \\
\hline SBSF 14 & 0767-0069418 & 060706.34 & -131337.1 & +12 & -26 & 9.8 & 9.0 & 7.99 & 56 & 20 & NVSS \\
\hline SBSF 15 & 0734-0203992 & 084400.22 & -163401.1 & -4 & 0 & 11.3 & 11.8 & 11.00 & 1 & 30 & \\
\hline SBSF 16 & 0705-0208711 & 091452.77 & -192617.0 & -12 & 0 & 11.0 & 11.2 & 10.89 & 1 & 30 & \\
\hline SBSF 17 & $0683-0253521$ & 094744.79 & -213712.7 & -8 & -12 & 12.0 & 11.6 & 10.70 & 1 & 30 & EIS D \\
\hline SBSF 18 & 0682-0261875 & 094946.99 & -214513.3 & 0 & 0 & 12.2 & 11.4 & 10.69 & 1 & 30 & EIS D \\
\hline SBSF 19 & $0682-0262596$ & 095144.77 & -214328.0 & -26 & +2 & 13.2 & 11.9 & 11.06 & 2 & 30 & EIS D \\
\hline SBSF 20 & 0696-0235495 & 095150.64 & -202014.8 & +8 & -34 & 12.4 & 12.4 & 10.46 & 2 & 30 & EIS D \\
\hline SBSF 21 & 0686-0238319 & 095232.44 & -212130.1 & -12 & +2 & 12.7 & 11.9 & 11.33 & 0 & 30 & EIS D \\
\hline SBSF 22 & 0696-0236655 & 095514.78 & -202003.3 & -12 & 0 & 12.4 & 10.6 & 10.23 & 1 & 30 & EIS D \\
\hline SBSF 23 & 0528-0285326 & 100922.50 & -371154.8 & -8 & 0 & 11.1 & 11.3 & 11.27 & 2 & 30 & \\
\hline SBSF 24 & 0599-0250386 & 104026.20 & -300036.5 & -14 & +8 & 11.2 & 11.6 & 11.40 & 2 & 30 & \\
\hline SBSF 25 & 0577-0367919 & 122402.32 & -321435.7 & -18 & -6 & 10.3 & 10.4 & 10.28 & 23 & 30 & \\
\hline SBSF 26 & 0593-0288091 & 124955.31 & -303818.7 & -26 & -2 & 10.4 & 10.4 & 10.00 & 23 & 30 & \\
\hline SBSF 27 & 0582-0321519 & 125537.48 & -314241.3 & -10 & -24 & 11.4 & 11.6 & 10.97 & 1 & 30 & \\
\hline SBSF 28 & 0478-0346964 & 125614.14 & -420910.9 & -8 & +4 & 10.4 & 10.5 & 10.79 & 23 & 30 & \\
\hline SBSF 29 & 0580-0344020 & 130318.33 & -315427.3 & -4 & -14 & 10.8 & 10.9 & 10.90 & 0 & 30 & \\
\hline SBSF 30 & 0550-0285689 & 130437.76 & -345630.8 & -6 & +6 & 11.4 & 11.7 & 10.80 & 1 & 30 & \\
\hline SBSF 31 & 0460-0297795 & 132832.45 & -435803.4 & -14 & -2 & 11.7 & 12.1 & 10.57 & 1 & 30 & \\
\hline SBSF 32 & $0562-0303721$ & 132859.77 & -334225.0 & -28 & +16 & 10.3 & 10.3 & 10.05 & 2 & 30 & \\
\hline SBSF 33 & 0568-0379911 & 134031.54 & -330752.6 & -12 & -16 & 10.7 & 10.8 & 10.76 & 2 & 30 & \\
\hline SBSF 34 & 0582-0339867 & 134625.24 & -314547.5 & +8 & -8 & 11.4 & 11.6 & 10.97 & 1 & 30 & \\
\hline SBSF 35 & $0745-0822065$ & 210025.58 & -152612.4 & -14 & -12 & 12.1 & 10.4 & 8.40 & 56 & 30 & NVSS \\
\hline SBSF 36 & 0615-0931505 & 221436.74 & -282531.6 & -16 & -16 & 11.7 & 10.6 & 9.36 & 5 & 30 & NVSS \\
\hline SBSF 37 & 0501-0829581 & 224304.43 & -394929.3 & +28 & -22 & 12.8 & 11.8 & 10.55 & 6 & 30 & EIS A \\
\hline SBSF 38 & 0498-0817047 & 224706.77 & -401001.3 & +18 & -8 & 11.5 & 10.8 & 9.29 & 6 & 30 & EIS A \\
\hline SBSF 39 & 0504-0833030 & 224934.23 & -393305.3 & +18 & -36 & 12.9 & 11.1 & 9.78 & 5 & 20 & EIS A \\
\hline SBSF 40 & 0501-0831035 & 224949.32 & -395315.0 & -36 & -28 & 12.4 & 11.8 & 10.64 & 5 & 30 & EIS A \\
\hline SBSF 41 & 0498-0817689 & 225021.28 & -400738.6 & +18 & 0 & 12.5 & 11.2 & 10.72 & 5 & 30 & EIS A \\
\hline SBSF 42 & 0714-0852904 & 232955.77 & -183554.1 & +6 & +4 & 11.0 & 9.6 & 7.90 & 6 & 30 & NVSS \\
\hline
\end{tabular}

3. Galactic latitude $|b| \geq 15^{\circ}$, in order to minimize extinction and contamination by large numbers of foreground stars; and

4. declination in the range $-44^{\circ} \leq \delta \leq-13^{\circ}$, so that AO observations from Cerro Paranal (at latitude $\sim-24^{\circ}$ ) can be obtained at low airmass.
Because the USNO-A2.0 catalogue's star-galaxy separation is not perfect (e.g., USNO-A2.0 0450-40377908 = ESO 436-G001), our search was not entirely automated. To check that favored objects were in fact stars, we used either the Tycho-2 catalog (Høg et al. 2000) or the publically available ESO Imaging Survey (EIS) data. Visual inspection of the 
Table 2. Log of observations.

\begin{tabular}{ccc}
\hline \hline $\begin{array}{c}\text { Night } \\
(1)\end{array}$ & $\begin{array}{c}\text { Date } \\
(2)\end{array}$ & $\begin{array}{c}\text { Conditions } \\
(3)\end{array}$ \\
\hline 1 & 2001 Feb. 15 & photometric \\
2 & 2001 Feb. 17 & photometric \\
3 & 2001 Mar. 07 & thin cirrus \\
4 & 2001 Oct. 06 & thin cirrus \\
5 & 2001 Oct. 07 & photometric \\
6 & 2001 Oct. 08 & photometric \\
\hline
\end{tabular}

I-band images for EIS Wide patches A (Nonino et al. 1999), B (Prandoni et al. 1999), and C and D (Benoist et al. 1999) allowed us to confirm that no additional bright stars or large foreground galaxies would block our view of more interesting background objects; we also tended to favor fields with one or more discernable I-band sources lying within $30^{\prime \prime}$ of the central stars. In a small number of cases, we relaxed our magnitude and color criteria to include brighter and redder stars located within $20^{\prime \prime}$ of radio sources detected in the NRAO VLA Sky Survey (NVSS: Condon et al. 1998). Our goal in imaging these fields (noted in the last column of Table 1) was to take advantage of the fact that radio galaxies often trace overdensities in the large-scale structure, seemingly at all redshifts (e.g., Hill \& Lilly 1991; Best 2000; Kurk et al. 2000; Pentericci et al. 2000; McLure \& Dunlop 2001). Subsequent to our observations, the release of the USNO-B1.0 catalogue ${ }^{1}$ (Monet et al. 2003) allowed us to verify that only one of the stars in our sam-

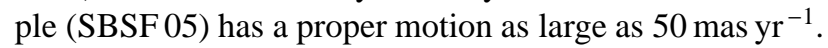

\subsection{Observations}

We obtained our observations using SOFI (Moorwood et al. 1998) on the ESO New Technology Telescope (NTT), over the course of six nights in February, March, and October 2001 (see Table 2). Conditions were photometric for two of the three nights in each season, allowing us to obtain good photometry for all of our fields; $K_{\mathrm{s}}$ seeing ranged from $0.5^{\prime \prime}$ to $1.0^{\prime \prime}$ with a median $\sim 0.7^{\prime \prime}$. Our choice of observing strategy was guided by our desire to optimize the detection of faint sources at small angular separations from the bright star in each field. We used SOFI's small-field objective lens, giving $0.145^{\prime \prime} \times 0.145^{\prime \prime}$ pixels and a $2.47^{\prime} \times 2.47^{\prime}$ field of view; larger pixels would only have hindered modelling of point spread function (PSF) wings close to the star, while a larger field of view would only have yielded additional sources too far from the star to be useful for future AO studies. In order to minimize memory effects in the $\mathrm{HgCdTe}$ array, we used short detector integration times (2-4 s, depending on the magnitude of the star) and spent a total of one minute on source before dithering the telescope to a new position. After imaging the field at ten such positions - each located randomly within a "jitter box" of dimensions 40 " $\times 40$ " we switched to a different bright star field for another ten-frame sequence. We would return to a particular field again later in

\footnotetext{
1 The preliminary photometry in the USNO-B1.0 catalogue shifts a few additional stars to $B-R>1.1$, as Table 1 indicates.
}

the same (or a different) night, in order to allow the diffraction spikes of the (Alt-Az mounted) NTT to rotate on the sky and thus expose any faint objects lying underneath. All of our fields were observed for a total of at least $20 \mathrm{~min}$, and most were observed for a total of $30 \mathrm{~min}$ (see Table 1). During each of the four photometric nights, we interspersed multiple observations of six or seven faint standard stars (Persson et al. 1998) in order to determine the flux scale.

\subsection{Data reduction}

We reduced the data using custom scripts within the NOAO IRAF package (Tody 1993). For each set of ten consecutive frames, we made an estimate of the (sky and dark current) background by masking out objects and - after further rejection of high and low pixels according to an estimated variance - taking a median. Following background subtraction, each frame was divided by a dome flat field (the difference of lamp on and lamp off frames) that had been corrected for the dependence of the array shade pattern on incident flux ${ }^{2}$. Values for bad pixels were then interpolated. We next addressed the problem of lowlevel array cross talk, which resulted from the bright star in the field and manifested as excess counts in two vertical stripes. To make a more precise measurement of this rather weak effect, we coarsely aligned the ten frames, averaged them, and measured the median of each column in the mean image. The resulting values were then subtracted from the corresponding columns in the individual unaligned frames. Finally, we performed a fine alignment to compensate for irregular behavior by the image derotator during our runs. Although the derotator always assumed the correct position for the initial frame in each set of ten, it often remained at the same position during the subsequent nine. We therefore rotated and translated frames $2-10$ in each set to align them with frame 1 . For the final image combination (of all 20-30 frames taken for each field), after rejection of high and low pixels according to an estimated variance, we calculated the mean of the remaining pixels at each point. The angular resolution in the final images was calculated as the FWHM of a Moffat (1969) profile fit to the central star in each field.

For each observing run separately, the conditions were sufficiently stable that we could use the same flux calibration scale for the entire dataset. The zero point magnitudes were $K_{\mathrm{s}}=$ $22.27 \pm 0.02$ for the February/March run and $K_{\mathrm{s}}=22.34 \pm 0.03$ for the October run, with uncertainties reflecting the scatter from multiple standard stars observed at a variety of times, airmasses, and positions on the detector during the course of each night. These and all other magnitudes in this paper are Vegarelative.

After measuring the $K_{\mathrm{s}}$ magnitudes of the central stars (see Table 1), we wanted - to the extent possible - to remove all traces of them from the images. To eliminate the (approximately) azimuthally symmetric part of each star's PSF, we fit ellipses to its isophotes. Inside the FWHM of the star's surface brightness profile, we allowed the position angle and

\footnotetext{
2 This type of "special" dome flat is described in Version 1.3 of the SOFI User's Manual.
} 
Table 3. Sources in bright star fields identified by SExtractor; columns are described in Sect. 3.1. A full version is available in electronic form at the CDS.

\begin{tabular}{|c|c|c|c|c|c|c|c|c|}
\hline $\begin{array}{c}\text { Source } \\
\text { identifier } \\
\text { (1) }\end{array}$ & $\begin{array}{c}\text { RA } \\
(\mathrm{J} 2000) \\
(2)\end{array}$ & $\begin{array}{c}\text { Dec } \\
(\mathrm{J} 2000) \\
(3)\end{array}$ & $\begin{array}{c}\Delta \theta \\
(\operatorname{arcsec}) \\
(4)\end{array}$ & $\begin{array}{c}K_{\mathrm{s}} \\
(\mathrm{mag}) \\
(5)\end{array}$ & $\begin{array}{c}\text { Major axis } \\
\text { (arcsec) } \\
(6)\end{array}$ & $\begin{array}{c}\text { Minor axis } \\
\text { (arcsec) } \\
(7)\end{array}$ & $\begin{array}{c}\text { PA } \\
\text { (degrees) } \\
(8)\end{array}$ & $\begin{array}{c}\text { Stellarity } \\
\text { index } \\
(9)\end{array}$ \\
\hline SBSF $01+077+024$ & 001139.38 & -193708.6 & 8.1 & 14.04 & 1.63 & 1.11 & 81 & 0.09 \\
\hline SBSF $01+047-093$ & 001139.17 & -193720.3 & 10.5 & 15.35 & 1.63 & 0.65 & 50 & 0.03 \\
\hline SBSF 01-132-076 & 001137.90 & -193718.6 & 15.2 & 20.12 & 0.14 & 0.11 & 28 & 0.42 \\
\hline SBSF $01+130+149$ & 001139.76 & -193656.1 & 19.8 & 18.60 & 0.35 & 0.29 & 71 & 0.96 \\
\hline SBSF 01-217-099 & 001137.30 & -193720.9 & 23.9 & 17.83 & 0.49 & 0.42 & 131 & 0.03 \\
\hline . & . & - & . & . & . & . & . & . \\
\hline . & . & . & . & . & . & . & . & . \\
\hline . & . & . & . & . & $\cdot$ & . & . & . \\
\hline SBSF 42-057-480 & 232955.36 & -183642.0 & 48.4 & 19.34 & 0.30 & 0.22 & 60 & 0.55 \\
\hline SBSF $42-487+056$ & 232952.34 & -183548.4 & 49.0 & 18.26 & 0.47 & 0.37 & 147 & 0.19 \\
\hline SBSF $42+516-130$ & 232959.40 & -183607.0 & 53.2 & 14.12 & 0.61 & 0.56 & 91 & 0.98 \\
\hline SBSF $42+335-466$ & 232958.12 & -183640.6 & 57.5 & 19.71 & 0.23 & 0.21 & 170 & 0.51 \\
\hline SBSF 42-016+590 & 232955.65 & -183455.0 & 59.0 & 20.82 & 0.12 & 0.10 & 47 & 0.38 \\
\hline
\end{tabular}

ellipticity to be free parameters; outside the FWHM, these were fixed to the values at the FWHM to permit isophote-fitting to the PSF wings out to a radius of $\sim 15^{\prime \prime}$. Deviating pixels (i.e., those that could in principle be due to faint underlying sources) were rejected in these fits. The azimuthally asymmetric diffraction spikes in the star's PSF had in general already been excised at the earlier stage of image combination, where their different orientations for the different sets of ten frames allowed them to be eliminated via high pixel rejection. For the small minority of fields where this was not possible or that centered on very bright stars, no satisfactory method could be found to remove the diffraction spikes that did not also compromise the quality of the data.

\section{Analysis}

\subsection{Object identification}

We used SExtractor (Bertin \& Arnouts 1996) to generate object catalogues from our final, PSF-subtracted images. Table 3 lists the sources, for each of which we provide the following information:

1. a unique identifier that combines a field name from Table 1 with the source's right ascension and declination offsets from the central star in tenths of arcseconds (SBSF 01+047-093, for example, is the object 4.7" east and 9.3" south of the bright star in field SBSF 01);

2. its right ascension, estimated from the right ascension offset and Cols. (3) through (5) of Table 1;

3. its declination, estimated from the declination offset and Cols. (4) and (6) of Table 1;

4. its angular separation from the bright star;

5. its $K_{\mathrm{s}}$ magnitude, using the SExtractor BEST definition that appears in simulations to be satisfactorily robust against aperture effects (Väisänen et al. 2000; cf. Martini 2001);

6. its semi-major axis, defined as the second-order moment (i.e., the intensity-weighted RMS) parallel to its major axis;
7. its semi-minor axis, defined as the second-order moment perpendicular to its major axis;

8. the position angle of its major axis, in degrees east of north; and

9. its SExtractor stellarity index, ranging from 0 (diffuse) to 1 (pointlike).

Within Table 3, the bright star fields are listed in order of right ascension; within each field, the individual sources are then listed in order of increasing angular separation from the central star, out to a maximum of $\Delta \theta=60^{\prime \prime}$. The full catalogue includes 1980 sources; this total does not include the 42 bright stars on which the fields themselves are centered.

We estimate that the offsets of each field's sources relative to the central star are accurate to within the size of a SOFI pixel, i.e., $\pm 0.075^{\prime \prime}$. Although this relative astrometry formally applies only to the 2001.1 and 2001.8 epochs of our observations, the nominal proper motions of the reference stars are small enough that (with the exception of SBSF 05) J2000.0 coordinates can essentially be computed from the field centers and source identifiers alone. We note that the absolute coordinates listed in Table 3 may be inaccurate if the central stars' true proper motions differ from the relative values listed in the USNO-B1.0 catalogue and Table 1, or if some of the (stellar) objects themselves have nonzero proper motions.

\subsection{Completeness and photometric accuracy}

We have used a single set of simulations to determine simultaneously the photometric accuracy and completeness limit as a function of magnitude for our full source catalogue. For each field, we extracted a $10^{\prime \prime} \times 10^{\prime \prime}$ cutout around every object with $K_{\mathrm{s}}<18$ and rescaled it by a constant factor so that the source magnitude was shifted into the range $16 \leq K_{\mathrm{s}}<21$. Brighter objects with true $K_{\mathrm{s}}<15$ (about half of which are the central stars themselves) were dimmed to magnitudes randomly distributed in the range $16 \leq K_{\mathrm{s}}<18$; fainter objects with true $15 \leq K_{\mathrm{s}}<18$ were dimmed to magnitudes randomly 
Table 4. Galaxy number counts as a function of $K_{\mathrm{S}}$ magnitude. Column (1) indicates the magnitude bin. Column (2) lists the corresponding completeness $f_{\mathrm{c}}$ (when reliable). Columns (3), (4), and (5) list the raw numbers of galaxies with source separations $\Delta \theta<5^{\prime \prime}, 5^{\prime \prime} \leq \Delta \theta<30^{\prime \prime}$, and $30^{\prime \prime} \leq \Delta \theta \leq 60^{\prime \prime}$, respectively. Columns (6), (7), and (8) list the completeness-corrected number counts of galaxies per magnitude per square degree for source separations $5^{\prime \prime} \leq \Delta \theta<30^{\prime \prime}, 30^{\prime \prime} \leq \Delta \theta \leq 60^{\prime \prime}$, and $5^{\prime \prime} \leq \Delta \theta \leq 60^{\prime \prime}$, respectively. We do not list number counts for the two faintest magnitude bins due to the substantial uncertainty in the true $f_{\mathrm{c}}$ at these levels. Uncertainties are calculated from Poissonian statistics following Gehrels (1986).

\begin{tabular}{cccccccc}
\hline \hline$K_{\mathrm{s}}$ & $f_{\mathrm{c}}$ & $N_{\text {raw }}(0,5)$ & $N_{\text {raw }}(5,30)$ & $N_{\text {raw }}(30,60)$ & $N(5,30)$ & $N(30,60)$ & $N(5,60)$ \\
$(1)$ & $(2)$ & $(3)$ & $(4)$ & $(5)$ & $(6)$ & $(7)$ & $(8)$ \\
\hline $12.5-13.5$ & 1.000 & 0 & 2 & 1 & $224_{-145}^{+297}$ & $36_{-30}^{+84}$ & $82_{-44}^{+80}$ \\
$13.5-14.0$ & 1.000 & 0 & 2 & 1 & $449_{-290}^{+592}$ & $73_{-61}^{+167}$ & $165_{-90}^{+160}$ \\
$14.0-14.5$ & 1.000 & 0 & 4 & 1 & $898_{-430}^{+710}$ & $73_{-61}^{+167}$ & $275_{-119}^{+185}$ \\
$14.5-15.0$ & 1.000 & 0 & 4 & 5 & $898_{-430}^{+110}$ & $364_{-157}^{+246}$ & $494_{-161}^{+226}$ \\
$15.0-15.5$ & 1.000 & 0 & 5 & 5 & $1122_{-484}^{+760}$ & $364_{-157}^{+246}$ & $549_{-170}^{+235}$ \\
$15.5-16.0$ & 1.000 & 0 & 7 & 10 & $1571_{-579}^{+847}$ & $728_{-227}^{+310}$ & $934_{-224}^{+286}$ \\
$16.0-16.5$ & 0.994 & 0 & 9 & 23 & $2033_{-665}^{+928}$ & $1684_{-349}^{+429}$ & $1769_{-311}^{+371}$ \\
$16.5-17.0$ & 0.990 & 1 & 15 & 28 & $3402_{-869}^{+124}$ & $2058_{-387}^{+466}$ & $2387_{-363}^{+422}$ \\
$17.0-17.5$ & 0.986 & 0 & 15 & 36 & $3415_{-872}^{+1130}$ & $2656_{-440}^{+521}$ & $2842_{-397}^{+457}$ \\
$17.5-18.0$ & 0.970 & 0 & 30 & 68 & $6943_{-1261}^{+1514}$ & $5100_{-616}^{+697}$ & $5552_{-560}^{+619}$ \\
$18.0-18.5$ & 0.958 & 1 & 54 & 80 & $12655_{-1716}^{+1968}$ & $6076_{-678}^{+758}$ & $7686_{-663}^{+723}$ \\
$18.5-19.0$ & 0.946 & 0 & 62 & 142 & $14714_{-1863}^{+2117}$ & $10921_{-915}^{+996}$ & $11849_{-829}^{+890}$ \\
$19.0-19.5$ & 0.888 & 0 & 69 & 188 & $17445_{-2094}^{+2364}$ & $15403_{-1122}^{+1208}$ & $15903_{-991}^{+1055}$ \\
$19.5-20.0$ & 0.717 & 0 & 78 & 235 & $24423_{-2758}^{+3091}$ & $23846_{-1554}^{+1659}$ & $23987_{-1355}^{+1434}$ \\
$20.0-20.5$ & 0.439 & 0 & 56 & 173 & $28638_{-3814}^{+4363}$ & $28672_{-2178}^{+2350}$ & $28664_{-1893}^{+2022}$ \\
$20.5-21.0$ & 0.127 & 1 & 32 & 114 & $56568_{-9952}^{+11880}$ & $65309_{-6107}^{+6709}$ & $63170_{-5223}^{+5672}$ \\
$21.0-21.5$ & - & 0 & 3 & 26 & - & - & - \\
$21.5-22.0$ & - & 0 & 1 & 2 & - & - & - \\
\hline
\end{tabular}

distributed in the range $16 \leq K_{\mathrm{s}}<21$, subject to the constraint of $\geq 1$ mag fading. We then pasted the rescaled cutout back into the original image at a random position with separation $5^{\prime \prime} \leq \Delta \theta \leq 55^{\prime \prime}$ from the central star. We did not consider test separations $\Delta \theta<5^{\prime \prime}$ due to the complex coupling of source detection with PSF subtraction at small radii (see e.g., Fig. 5), but avoided separations $55^{\prime \prime}<\Delta \theta \leq 60^{\prime \prime}$ merely to minimize edge effects. The enforcement of $\geq 1$ mag fading ensured that the noise would remain identical to that in the original image. Using the same parameters as in the original execution, we ran SExtractor on the modified image and attempted to recover the fictitious object within $0.5^{\prime \prime}$ of its nominal position. The procedure was repeated 100 times for every $K_{\mathrm{s}}<18$ object in each of the 42 fields, giving a total of 57,800 iterations.

Figure 2 and Table 4 show the completeness we derive over the range $16 \leq K_{\mathrm{s}}<21$ from the dataset as a whole, i.e., treating the sum of our $425^{\prime \prime} \leq \Delta \theta \leq 60^{\prime \prime}$ annuli as the "discrete deep field" equivalent of a single contiguous $131 \mathrm{arcmin}^{2}$ area with patchy sensitivity. We estimate our catalogue is $90 \%$ complete to $K_{\mathrm{s}}=19.2$ and $50 \%$ complete to $K_{\mathrm{s}}=20.2$. These values include the effects of non-detections due to the proximity of another (brighter) object, typically $1-2 \%$ at $K_{\mathrm{S}} \sim 17$, and apply as well to extended as to point sources. The error bars in Fig. 2 indicate the field-to-field variations in completeness, which result from differences in integration time, seeing, and source surface density.

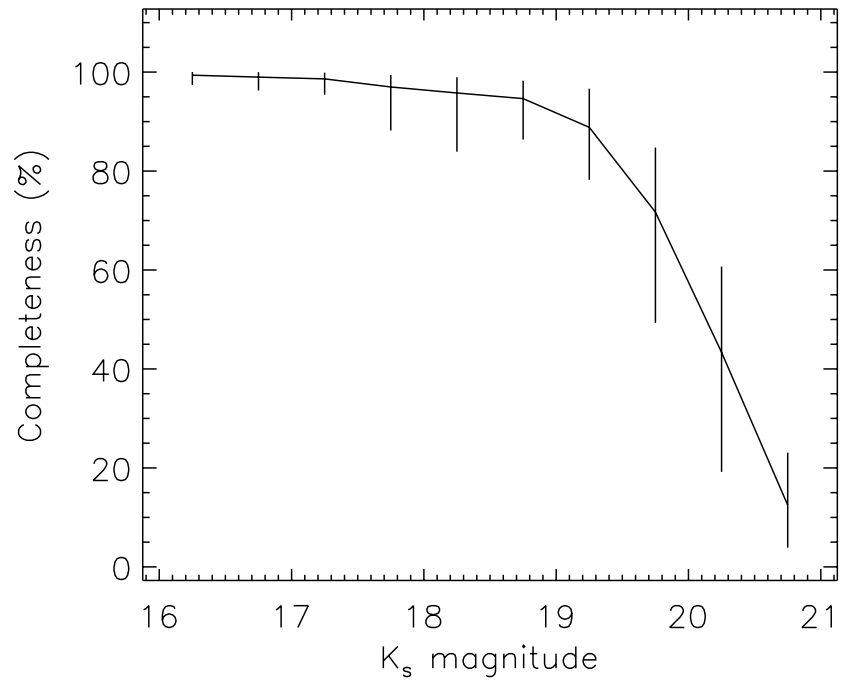

Fig. 2. Completeness as a function of magnitude. The solid line is the mean over all 42 fields; the error bars indicate the dispersions between fields, reflecting field-to-field variations in sensitivity.

Because SExtractor calculates a magnitude for each of the fictitious sources it recovers in the 57,800 simulated images described above, we can estimate the uncertainty in our photometry from the distribution of differences between the recovered sources' nominal and measured magnitudes 


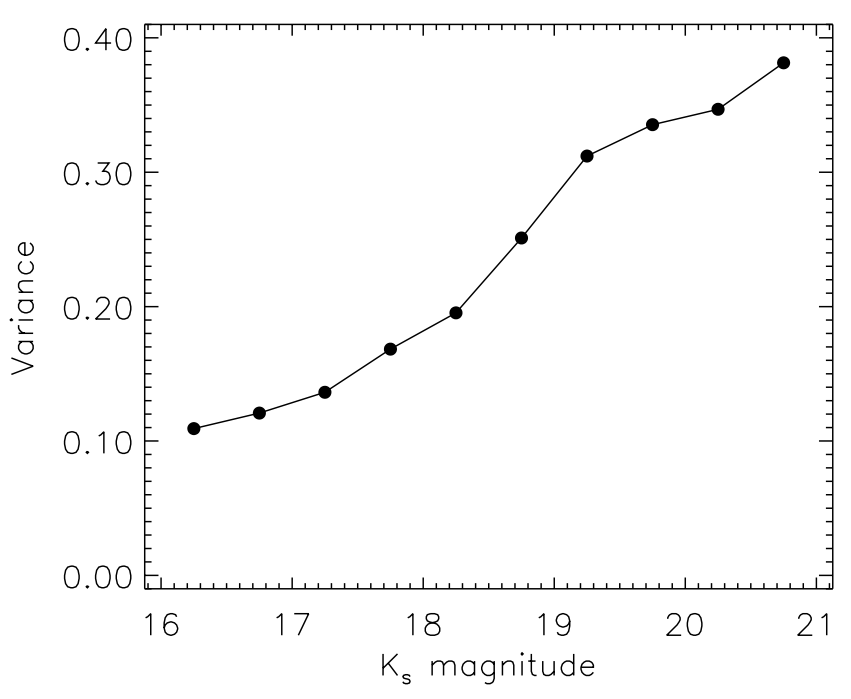

Fig. 3. Uncertainty in our estimate of $K_{\mathrm{s}}$ magnitude as a function of true source magnitude. As described in Sect. 3.2, we plot the variance of the distribution of measured magnitudes for each set of nominal magnitudes (i.e., of fictitious objects pasted into simulated images).

$\left(\Delta m \equiv m_{\text {meas }}-m_{\text {nom }}\right)$. Empirically, this distribution is symmetric about zero (i.e., $\left\langle\Delta m>=0\right.$ ) down to $K_{\mathrm{s}}=20$ with large non-Gaussian wings. At fainter levels, $<\Delta m>$ begins to skew negative as sources with $m_{\text {meas }}>m_{\text {nom }}$ become increasingly difficult for SExtractor to recover. Figure 3 plots the variance $<\Delta m^{2}>-<\Delta m>^{2}$ (after rejection of a few catastrophic outliers) as a function of $m_{\text {nom }}$. The implied errors are significantly larger than the formal uncertainties returned by SExtractor, and provide a more realistic indication of the true accuracy of our photometry.

\subsection{Galaxy counts vs. $K_{\mathrm{s}}$ magnitude}

In order to evaluate the reliability of our source catalogue, we have derived a magnitude-counts relation from our data suitable for comparison with the published literature. We begin by using the SExtractor stellarity index to perform star/galaxy separation within our source catalogue. In addition to the $240 \mathrm{ob}-$ jects with stellarity index $>0.97$ that are clearly stars, we exclude an additional 151 sources with stellarity index above a more conservative threshold of 0.90 . This leaves 1589 sources in the 42 catalogue fields, of which 3 lie at separations $\Delta \theta<5^{\prime \prime}$, 448 at $5^{\prime \prime} \leq \Delta \theta<30^{\prime \prime}$, and 1138 at $30^{\prime \prime} \leq \Delta \theta \leq 60^{\prime \prime}$. Division into these three subsets isolates the ranges of source separation in which the simulations of Sect. 3.2 do not constrain the completeness (i.e., $\Delta \theta<5^{\prime \prime}$ ), and in which particularly special care will be needed to model AO PSF variation as a function of radius (i.e., $30^{\prime \prime} \leq \Delta \theta \leq 60^{\prime \prime}$; see Steinbring et al. 2002). Table 4 bins the data by magnitude, and for the range over which we have been able to make reliable completeness corrections $\left(12.5 \leq K_{\mathrm{s}}<21\right)$ lists the differential number counts from both of the outer annuli and the full $5^{\prime \prime} \leq \Delta \theta \leq 60^{\prime \prime}$ range, normalized to units of counts per magnitude per square degree. The $1 \sigma$ error bars on the normalized counts are derived from Poissonian statistics according to the prescriptions of Gehrels (1986).

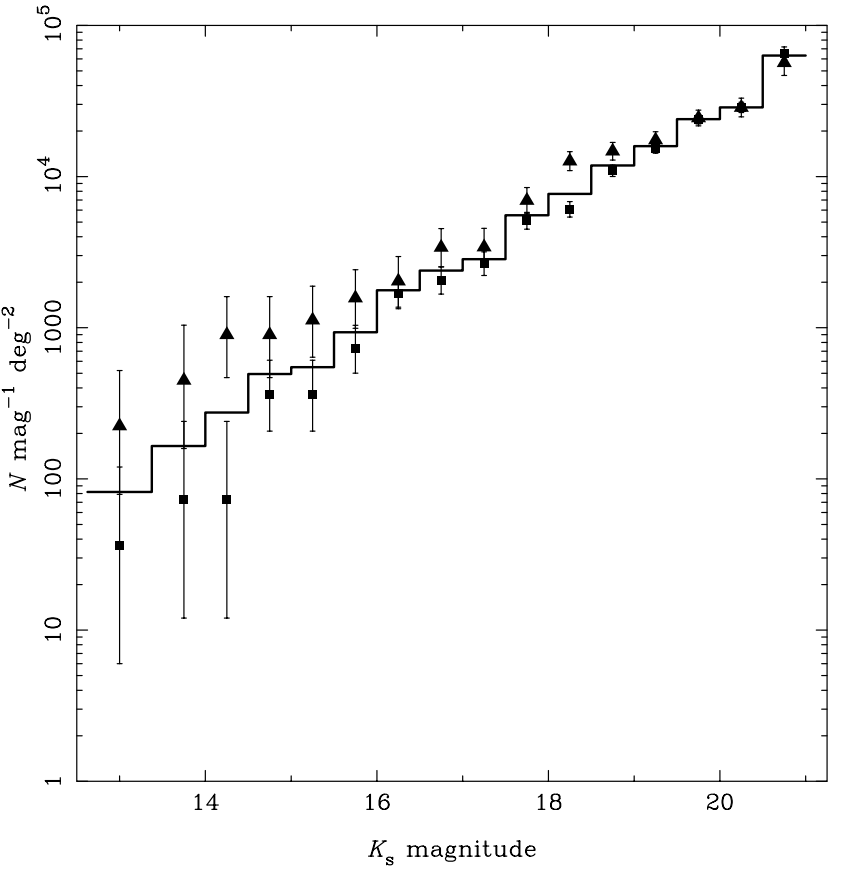

Fig. 4. Differential galaxy number counts as a function of $K_{\mathrm{s}}$ magnitude. Triangles and squares indicate completeness-corrected counts for the disjoint sets of sources with separations $5^{\prime \prime} \leq \Delta \theta<30^{\prime \prime}$ and $30^{\prime \prime} \leq \Delta \theta \leq 60^{\prime \prime}$ from the bright stars (i.e., Cols. (6) and (7) in Table 4), respectively. The thick solid line shows the relation (also completeness-corrected) when these two subsets are combined (Col. (8) in Table 4).

Figure 4 plots the completeness-corrected counts for the two annuli (as discrete points with error bars) and for the full sample (as a continuous curve). It is immediately apparent as from comparison of Cols. (6) and (7) in Table 4 - that our 42 fields in aggregate have a higher surface density of background sources at separations $5^{\prime \prime} \leq \Delta \theta<30^{\prime \prime}$ from the star than at separations $30^{\prime \prime} \leq \Delta \theta \leq 60^{\prime \prime}$ in the brighter magnitude bins. This result is not surprising, given that a number of our fields were selected in part precisely because they did reveal background sources within $30^{\prime \prime}$ of the central stars in I-band images. In particular, when we compare plots of mean galaxy surface density versus angular separation for 23 fields in EIS Wide patches (most subject to this bias) and for 14 fields not in EIS Wide patches or near NVSS sources, as in the two panels of Fig. 5, we see a slight step in surface density at $30^{\prime \prime}$ in the former plot that is absent from the latter ${ }^{3}$. Note that this effect arises entirely in the bright magnitude bins; at fainter magnitudes $\left(K_{\mathrm{s}} \geq 19\right)$, Table 4 and Fig. 4 indicate that there is no gradient in the source density as a function of source separation. At the still fainter magnitudes that can only be reached with $\mathrm{AO}$-assisted imaging of these fields, there should again be no dependence of source surface density on separation from the bright star.

\footnotetext{
3 The slight difference in mean surface densities between EIS $\left(11.3 \mathrm{arcmin}^{-2}\right)$ and non-EIS (13.9 $\left.\mathrm{arcmin}^{-2}\right)$ fields may be due to residual contamination by faint stars in the latter, which tend to lie at somewhat lower Galactic latitudes.
} 

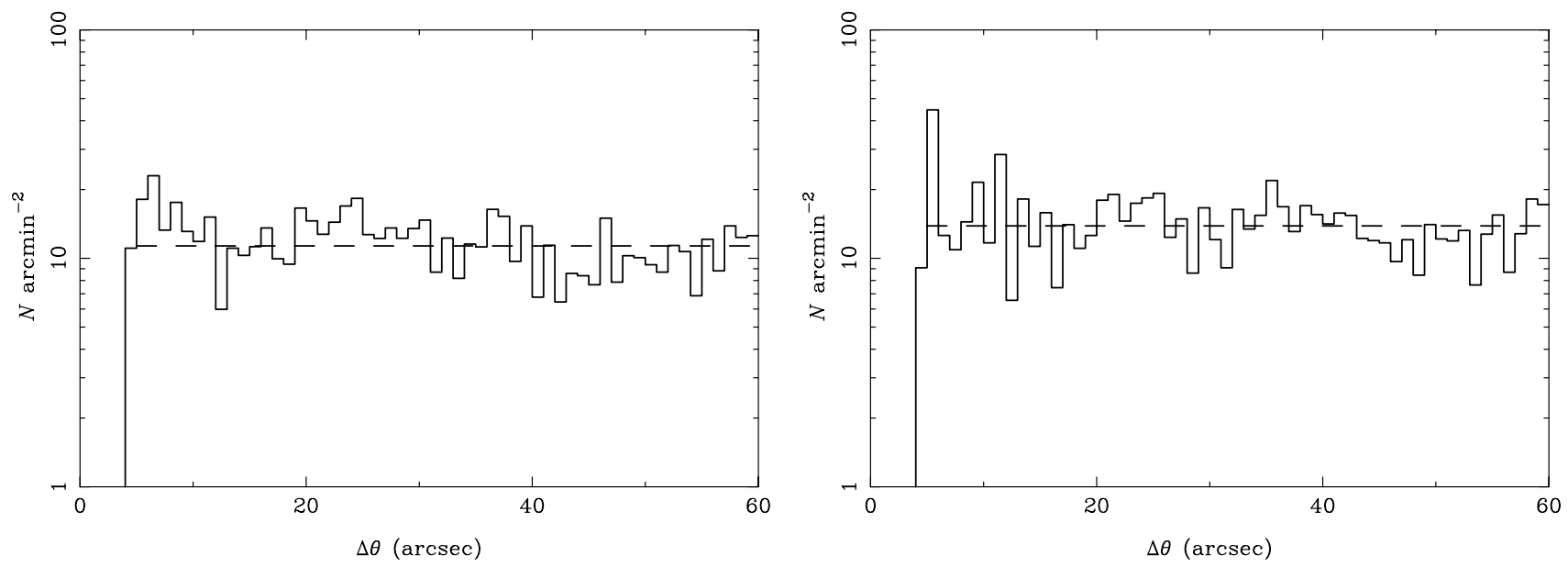

Fig. 5. Galaxy surface densities as a function of separation from the bright star in our non-NVSS fields. Left panel shows mean for 23 fields within EIS Wide patches; right panel shows mean for 14 fields outside of EIS Wide patches. Only for $\Delta \theta<5^{\prime \prime}$ does the star have an effect on the detectability of other objects. Dashed lines indicate the average number densities for $5^{\prime \prime} \leq \Delta \theta \leq 60^{\prime \prime}$. The slight increase in number density at $\Delta \theta<30^{\prime \prime}$ for the EIS fields results from our having preferentially imaged fields with one or more detected $I$-band sources at $\Delta \theta<30^{\prime \prime}$.

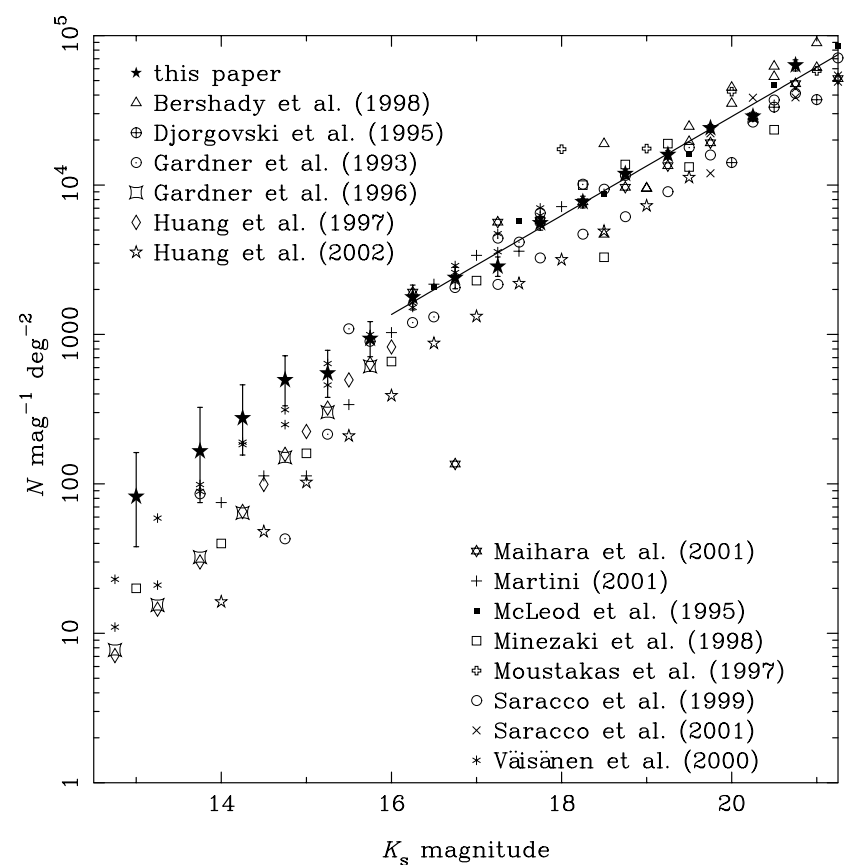

Fig. 6. Differential galaxy number counts as a function of $K_{\mathrm{s}}$ magnitude, compared to results from the literature. The solid line indicates the best power-law fit to our data (stars with error bars) for $16 \leq K_{\mathrm{s}}<21$ and $5^{\prime \prime} \leq \Delta \theta \leq 60^{\prime \prime}$ and has a slope $\alpha=0.33 \pm 0.01$. References for other symbols are as indicated in the figure.

Figure 6 shows a comparison of the completeness-corrected galaxy number counts calculated from the full $5^{\prime \prime} \leq \Delta \theta \leq 60^{\prime \prime}$ sample with comparable results from the literature. This figure includes a line showing the best power-law fit to the number counts for the range $16 \leq K_{\mathrm{s}}<21$; we derive a slope $\alpha \equiv$ $\mathrm{d} \log N / \mathrm{d} m=0.33 \pm 0.01$. Leaving out the $K_{\mathrm{s}} \sim 20.75$ point, which has a large and uncertain correction for incompleteness, gives $\alpha=0.31 \pm 0.01$; recomputing the counts from the 37 nonNVSS fields only (to circumvent the plausible clustering of the NVSS fields' faint sources) gives $\alpha=0.34 \pm 0.01$. In all cases, the agreement with previously published values in the range 0.32-0.37 is excellent (e.g., Djorgovski et al. 1995; McLeod et al. 1995; Bershady et al. 1998; McCracken et al. 2000; Huang et al. 2001). Our number counts are also in good agreement with prior results in terms of normalization for $K_{\mathrm{s}} \geq 16$ (although brighter magnitude bins are affected by the selection bias discussed above). The overall agreement with the literature gives us confidence that our approach to source identification is sensible, and (for faint magnitudes) that our fields define a representative slice of the extragalactic $2.2 \mu \mathrm{m}$ sky.

\section{Conclusions}

The agreement of the magnitude-counts relation derived from analysis of our "discrete deep field" with previous derivations by other authors is not a great surprise. Indeed, we would expect a priori that our results should be no more biased by cosmic variance than those of groups working with contiguous fields of equivalent total area, although the latter will be more useful for direct measurements of clustering strengths. The consistency demonstrates that our somewhat ad hoc approach to field selection - with some dependence on the availability of EIS data and/or the proximity of a bright radio or optical source - has not yielded a biased picture of the deep $2.2 \mu \mathrm{m}$ sky. As a result, it is possible to proceed to AO observations of this set of fields with full confidence that any conclusions based on the aggregate properties of their faint near-IR sources will be statistically sound. We hope that the southern hemisphere user community will find the catalogue of 391 faint stars and 1589 galaxies we have provided here to be useful in fully exploiting the use of $8 \mathrm{~m}$-class telescopes at or near their diffraction limits.

Acknowledgements. We thank the staff at the NTT, in particular support astronomers Stéphane Brillant and Pierre Leisy, for their advice on strategy and help in conducting the observations. We are grateful to João Alves and Charlie Lada for executing the 7 March 2001 
observations on our behalf as part of a time swap, and to Hélène Dickel and James Larkin for helpful suggestions. M. J. J. has been supported by the European Research and Training Network on the Physics of the Intergalactic Medium. This research has made use of NASA's Astrophysics Data System Bibliographic Services, and of the NASA/IPAC Extragalactic Database (NED), which is operated by the Jet Propulsion Laboratory, California Institute of Technology, under contract with NASA.

\section{References}

Benoist, C., da Costa, L., Olsen, L. F., et al. 1999, A\&A, 346, 58 Bershady, M. A., Lowenthal, J. D., \& Koo, D. C. 1998, ApJ, 505, 50 Bertin, E., \& Arnouts, S. 1996, A\&AS, 117, 393

Best, P. N. 2000, MNRAS, 317, 720

Condon, J. J., Cotton, W. D., Greisen, E. W., et al. 1998, AJ, 115, 1693

Davies, R. I., Lehnert, M., Baker, A. J., \& Rabien, S. 2001, in Galaxies and their Constituents at the Highest Angular Resolution, ed. R. T. Schilizzi, S. Vogel, F. Paresce, \& M. Elvis (San Francisco: ASP), 455

Davies, R., Lehnert, M., Baker, A. J., et al. 2003, in Discoveries and Research Prospects from 6- to 10-Meter-Class Telescopes II, ed. P. Guhathakurta, Proc. SPIE, 4838, 302

Djorgovski, S., Soifer, B. T., Pahre, M. A., et al. 1995, ApJ, 438, L13

Epchtein, N., de Batz, B., Capoani, L., et al. 1997, ESO Messenger, 87,27

Gardner, J. P., Cowie, L. L., \& Wainscoat, R. J. 1993, ApJ, 415, L9

Gardner, J. P., Sharples, R. M., Carrasco, B. E., \& Frenk, C. S. 1996, MNRAS, 282, L1

Gehrels, N. 1986, ApJ, 303, 336

Glassman, T. M., Larkin, J. E., \& Lafreniére, D. 2002, ApJ, 581, 865

Hill, G. J., \& Lilly, S. J. 1991, ApJ, 367, 1

Høg, E., Fabricius, C., Makarov, V. V., et al. 2000, A\&A, 357, 367

Huang, J.-S., Cowie, L. L., Gardner, J. P., et al. 1997, ApJ, 476, 12

Huang, J.-S., Thompson, D., Kümmel, M. W., et al. 2001, A\&A, 368, 787

Kurk, J. D., Röttgering, H. J. A., Pentericci, L., et al. 2000, A\&A, 358, L1

Larkin, J. E., \& Glassman, T. M. 1999, PASP, 111, 1410

Larkin, J. E., Glassman, T. M., Wizinowich, P., et al. 2000, PASP, 112, 1526

Le Louarn, M., Foy, R., Hubin, N., \& Tallon, M. 1998, MNRAS, 295, 756
Lenzen, R., Hofmann, R., Bizenberger, P., \& Tusche, A. 1998, Proc. SPIE, 3354, 606

Maihara, T., Iwamuro, F., Tanabe, H., et al. 2001, PASJ, 53, 25

Martini, P. 2001, AJ, 121, 2301

McCracken, H. J., Metcalfe, N., Shanks, T., et al. 2000, MNRAS, 311, 707

McLeod, B. A., Bernstein, G. M., Rieke, M. J., Tollestrup, E. V., \& Fazio, G. G. 1995, ApJS, 96, 117

McLure, R. J., \& Dunlop, J. S. 2001, MNRAS, 321, 515

Minezaki, T., Kobayashi, Y., Yoshii, Y., \& Peterson, B. A. 1998, ApJ, 494, 111

Moffat, A. F. J. 1969, A\&A, 3, 455

Monet, D., Bird A., Canzian, B., et al. 1998, VizieR Online Data Catalog, I/252

Monet, D. G., Levine, S. E., Canzian, B., et al. 2003, AJ, 125, 984 (also: VizieR Online Data Catalog, I/284)

Moorwood, A., Cuby, J.-G., \& Lidman, C. 1998, ESO Messenger, 91,

Moustakas, L. A., Davis, M., Graham, J. R., et al. 1997, ApJ, 475, 445

Nonino, M., Bertin, E., da Costa, L., et al. 1999, A\&AS, 137, 51

Pentericci, L., Kurk, J. D., Röttgering, H. J. A., et al. 2000, A\&A, 361, L25

Persson, S. E., Murphy, D. C., Krzeminski, W., Roth, M., \& Rieke, M. J. 1998, AJ, 116, 2475

Prandoni, I., Wichmann, R., da Costa, L., et al. 1999, A\&A, 345, 448

Rousset, G. 1994, in Adaptive Optics for Astronomy, ed. D. Alloin \& J. M. Mariotti (Dordrecht: Kluwer), 115

Rousset, G., Lacombe, F., Puget, P., et al. 1998, Proc. SPIE, 3353, 508

Saracco, P., D’Odorico, S., Moorwood, A., et al. 1999, A\&A, 349, 751

Saracco, P., Giallongo, E., Cristiani, S., et al. 2001, A\&A, 351, 1

Skrutskie, M. F., Schneider, S. E., Stiening, R., et al. 1997, in The Impact of Large Scale Near-IR Sky Surveys, ed. F. Garzón, N. Epchtein, A. Omont, W. B. Burton, \& P. Persi (Dordrecht: Kluwer), 25

Steinbring, E. L., Faber, S. M., Hinkley, S., et al. 2002, PASP, 114, 1267

Tallon, M., \& Foy, R. 1990, A\&A, 235, 549

Thatte, N. A., Tecza, M., Eisenhauer, F., et al. 1998, Proc. SPIE, 3353, 704

Tody, D. 1993, in Astronomical Data Analysis Software and Systems II, ed. R. J. Hanisch, R. J. V. Brissenden, \& J. Barnes (San Francisco: ASP), 173

Väisänen, P., Tollestrup, E. V., Willner, S. P., \& Cohen, M. 2000, ApJ, 540,593 